\title{
Degradation of Chondroitin Sulfate Proteoglycans Induces Sprouting of Intact Purkinje Axons in the Cerebellum of the Adult Rat
}

\author{
Luigi Corvetti and Ferdinando Rossi \\ Department of Neuroscience and Rita Levi Montalcini Center for Brain Repair, University of Turin, I-10125 Turin, Italy
}

\begin{abstract}
Chondroitin sulfate proteoglycans are major constituents of the extracellular matrix and form perineuronal nets. Information regarding the growth-inhibitory activity of these molecules after injury is rapidly expanding. However, less is known about their physiological role in the adult undamaged CNS. Here, we investigated the function of chondroitin sulfate proteoglycans in maintaining the proper structure of Purkinje axons in the cerebellum of adult rats. To this end, we examined the morphology and distribution of intracortical Purkinje neurites after intraparenchymal injection of chondroitinase ABC. Staining with the lectin Wisteria floribunda agglutinin or 2B6 antibodies showed that this treatment efficiently removed chondroitin sulfate proteoglycans from wide areas of the cerebellar cortex. In the same sites, there was a profuse outgrowth of terminal branches from the Purkinje infraganglionic plexus, which invaded the deeper regions of the granular layer. In contrast, myelinated axon segments were not affected and maintained their normal relationship with oligodendroglial sheaths. Purkinje axon sprouting was first evident at $4 \mathrm{~d}$ and increased further at $7 \mathrm{~d}$ after enzyme application. Within $42 \mathrm{~d}$, the expression pattern of chondroitin sulfate proteoglycans gradually recovered, whereas axonal modifications progressively regressed. Our results show that, in the absence of injury or novel external stimuli, degradation of chondroitin sulfate proteoglycans is sufficient to induce Purkinje axon sprouting but not the formation of long-lasting synaptic contacts. Together with other growth-inhibitory molecules, such as myelin-associated proteins, chondroitin sulfate proteoglycans restrict structural plasticity of intact Purkinje axons to maintain normal wiring patterns in the adult cerebellar cortex.
\end{abstract}

Key words: axon growth inhibitory molecules; axon regeneration; structural plasticity; extracellular matrix; myelin; Purkinje cell

\section{Introduction}

A fine regulation of neuritic growth is required in the adult CNS to preserve specific connections while allowing structural plasticity. This function is primarily mediated by two sets of molecules, which include several myelin-associated proteins [Nogo-A, MAG, Omgp (oligodendrocyte-myelin glycoprotein), Sema4D (semaphorin 4D)] (Filbin, 2003; Moreau-Fauvarque et al., 2003; Schwab, 2004) and glial scar components, such as chondroitin sulfate proteoglycans (CSPGs) (Fawcett and Asher, 1999; Silver and Miller, 2004). Indeed, although these molecules are primarily known for hampering axon regeneration after injury, they are widely expressed in the intact brain, in which they may exert a constitutive regulatory action on physiological neuritic growth and plasticity.

Proteoglycans are present in the extracellular matrix of differ-

Received Feb. 19, 2005; revised June 16, 2005; accepted June 17, 2005

This work was supported by grants from the International Institute for Research in Paraplegia (Zurich, Switzerland; P81/04), the Ministero dell'Università e della Ricerca Scientifica e Tecnologica (FIRB-RBNE01YRA3-004), and the University of Turin. We are indebted to Dr. Piergiorgio Strata for the use of the confocal microscope and to Dr. Ian Martin Williams for revising English style. We also thank Luisella Milano and Dr. Francesco Bertolo for precious technical assistance.

Correspondence should be addressed to Luigi Corvetti, Rita Levi-Montalcini Center for Brain Repair, Department of Neuroscience, Section of Physiology, University of Turin, Corso Raffaello 30, I-10125 Turin, Italy. E-mail: luigi.corvetti@unito.it.

DOI:10.1523/JNEUROSCI.0683-05.2005

Copyright $\odot 2005$ Society for Neuroscience $\quad$ 0270-6474/05/257150-09\$15.00/0 ent tissues. They consist of a protein core linked by sugar moieties to glycosaminoglycan (GAG) chains, which mediate functional interactions with cell-surface receptors or other components of the extracellular matrix (Bandtlow and Zimmermann, 2000). In the CNS, the most abundant proteoglycans are those containing sulfated GAG chains (Bandtlow and Zimmermann, 2000). In particular, several types of CSPGs are exclusively expressed in the nervous tissue and are part of the perineuronal net (Brauer et al., 1993; Bertolotto et al., 1996). During neural development, CSPGs regulate axon growth and pathfinding (Snow et al., 1990; Jhaveri, 1993). After injury in the adult, they are enriched in scar tissues (McKeon et al., 1991; Levine, 1994; Lemons et al., 1999; Properzi et al., 2005), in which they contribute to hampering axon regeneration (Davies et al., 1997; Moon et al., 2001; Bradbury et al., 2002; Grimpe and Silver, 2002; Jones and Tuszynski, 2002) or compensatory sprouting (Tropea et al., 2003). In addition, several studies have highlighted their role in controlling structural plasticity in the absence of injury (Brakebusch et al., 2002; Pizzorusso et al., 2002; Miyata et al., 2004). Together, these reports indicate that CSPGs regulate the growth of intact neurites, but the actual pattern of sprouting has not been demonstrated.

To address this issue, we examined the infraganglionic plexus of Purkinje axons. In the adult cerebellar cortex, the plexus is typically confined to the most superficial region of the granular layer (Ramón y Cajal, 1911). Procedures that disrupt myelino- 
genesis or application of neutralizing antibodies against Nogo-A induce aberrant sprouting from Purkinje axons that invade the deep portions of the granular layer. This indicates that the peculiar distribution pattern of the intracortical plexus is maintained, at least in part, by inhibitory signals issued by myelin and/or associated proteins (Buffo et al., 2000; Gianola et al., 2003). Because different types of CSPGs are strongly expressed in the adult cerebellum (Rauch et al., 1991; Yamada et al., 1997), here we asked whether these molecules also participate in this regulation. Thus, we induced degradation of CSPGs by intraparenchymal injection of chondroitinase $\mathrm{ABC}$ (ChABC) and examined the morphology and distribution of intracortical Purkinje axons. The profuse sprouting observed after CSPG removal indicates that these molecules actively restrict Purkinje axon plasticity to maintain their normal distribution pattern in the adult cerebellar cortex.

\section{Materials and Methods}

Animals and surgical procedures. Experiments were conducted on adult Wistar rats weighing 200-250 g (Charles River Italia, Calco, Italy). All surgical procedures were performed under deep general anesthesia induced by intraperitoneal administration of a mixture of ketamine and xylazine (100 mg/kg Ketalar and 5 mg/kg Rompun; Bayer, Leverkusen, Germany). The experimental plan was designed according to the European Communities Council Directive of 1986 (86/609/EEC), the National Institutes of Health guidelines, and the Italian law for care and use of experimental animals (DL116/92) and approved by the Italian Ministry of Health.

The enzymatic degradation of CSPGs was achieved by injection of ChABC, which catalyzes the removal of chondroitin sulfate and dermatan sulfate side chains of proteoglycans, yielding a protein core and carbohydrate stubs (Caterson et al., 1985). Animals were deeply anesthetized and fixed on a stereotaxic apparatus. Small pieces of the occipital bone were carefully removed to expose the posterior vermal lobules. Protease-free ChABC (Seikagaku, Tokyo, Japan) or saline vehicle only was injected in the cerebellar parenchyma. One microliter injections of a $24 \mathrm{U} / \mathrm{ml}$ solution of the enzyme were placed $1 \mathrm{~mm}$ deep into three adjacent lobules along the midline of the cerebellar vermis by means of a glass pipette mounted on a three-axis micromanipulator connected to a PV800 pneumatic picopump (World Precision Instruments, New Haven, CT). After surgery, the animals were kept in normal housing conditions for different survival periods of 1, 4, 7, 21, and $42 \mathrm{~d}$. Five enzymetreated and five vehicle-injected rats were examined at each time point, except at $7 \mathrm{~d}$ when six animals per group were considered.

Histological procedures. At the end of the survival period, the rats were deeply anesthetized and transcardially perfused with $500 \mathrm{ml}$ of $4 \%$ paraformaldehyde in $0.12 \mathrm{M}$ phosphate buffer, $\mathrm{pH}$ 7.2-7.4. The brains were immediately dissected, postfixed in the same fixative overnight at $4^{\circ} \mathrm{C}$, and transferred to $30 \%$ sucrose in phosphate buffer at $4^{\circ} \mathrm{C}$ until they sank. The cerebellum was isolated and cut by means of a freezing microtome in four series of $30-\mu \mathrm{m}$-thick sagittal sections, collected in Trisbuffered saline (TBS), $\mathrm{pH} 7.4$.

Section series that were processed for diaminobenzidine (DAB) staining were first incubated $20 \mathrm{~min}$ in $0.3 \%$ hydrogen peroxide in TBS to quench endogenous peroxidase. To evaluate the distribution of CSPGs within the cerebellar lobules, one series was incubated overnight at room temperature with biotinylated Wisteria floribunda agglutinin (WFA; 1:1000; Sigma, St. Louis, MO). The reaction was visualized with a standard avidin-biotin peroxidase method (Vectastain; ABC Elite kit; Vector Laboratories, Burlingame, CA) and revealed using nickel-enhanced DAB as a chromogen.

All of the primary and secondary antibodies used for immunocytochemical staining were diluted in PBS plus $0.25 \%$ Triton X-100 (PBST), containing $2 \%$ of either normal goat serum or horse serum, depending on the species of the second antibody used. To stain Purkinje cells, sections were incubated overnight at room temperature with the polyclonal anti-calbindin D-28K (1:3000; Swant, Bellinzona, Switzerland) and then
$1 \mathrm{~h}$ with the biotinylated goat anti-rabbit IgG. After washing, the reaction was revealed with the $\mathrm{ABC}$ method (as above) using $\mathrm{DAB}$ as a chromogen. Reacted sections were mounted on chrome-alum-subbed slides, air-dried, dehydrated, and coverslipped with Neo-Entellan (Merck, Darmstadt, Germany).

The effectiveness of ChABC injection was evaluated using the monoclonal antibody 2B6, which binds a four-sugar stub left behind CSPGs after chondroitinase digestion (Moon et al., 2001). Sections were incubated overnight with the monoclonal antibody 2B6 (1:200; Seikagaku) and then for $1 \mathrm{~h}$ with fluorescein isothiocyanate (FITC)-conjugated secondary antibody (1:200 in PBST with normal horse serum; Sigma).

Double immunofluorescence was used to study the relationship between Purkinje cells and either CSPGs or myelin. To reveal myelin, sections were first quenched in a solution of hydrogen peroxide (1:100) in methanol $20 \mathrm{~min}$ at room temperature. After overnight incubation with the polyclonal anti-calbindin antibody (1:1500; Swant) and $1 \mathrm{~h}$ incubation in FITC-conjugated goat anti-rabbit IgG (1:200 in PBST with normal goat serum; Sigma), sections were washed, exposed overnight to the monoclonal anti-myelin basic protein antibody (MBP; 1:1000; Sternberger Monoclonals, Lutherville, MD), and then exposed for $1 \mathrm{~h}$ with a secondary Texas Red-conjugated horse anti-rabbit IgG (1:200; Vector Laboratories). For double staining of Purkinje cells and CSPGs, the sections were first incubated overnight at room temperature in a mixture containing both monoclonal anti-parvalbumin antibody (1:400; Swant) and WFA (1:200; Sigma) and then revealed by $1 \mathrm{~h}$ incubation with a second mixture containing Texas Red-conjugated horse anti-mouse IgG (1:200; Vector Laboratories) and FITC-conjugated streptavidin (1:50; Sigma). Fluorescent sections were left to dry and coverslipped using Mowiol (Calbiochem, San Diego, CA).

Qualitative and quantitative analysis. The histological preparations were examined using a Zeiss (Oberkochen, Germany) Axiophot light microscope. Micrographs were taken with a Nikon (Tokyo, Japan) DS-5M camera head connected to the same microscope. The material was also examined with a Fluoview 300 confocal microscope (Olympus Optical, Hamburg, Germany).

Quantitative morphometric evaluations were made according to previously established procedures (Buffo et al., 2000), using Neurolucida software (MicroBrightField, Colchester, VT) connected to a Nikon E-800 microscope via a color CCD camera. Purkinje axons were evaluated at different survival times after enzyme or vehicle application (see above) on DAB-processed calbindin-immunolabeled cerebellar sections (see examples in Fig. $4 A-D$ ). All of the measurements were performed by the same observer (L.C.), who was unaware of the treatment received by the case under examination. To sample axons from the same cortical region in all of the animals, we restricted our analysis to vermal sections close to the midline and adjacent to the injection site (sections containing the pipette track were excluded to rule out possible effects induced by direct mechanical injury). On such sections, a $250 \times 150 \mu \mathrm{m}$ large area was sampled from the distal portion of lobule VI under a $40 \times$ objective. The selected area encompassed the entire granular layer depth and contained only minimal portions of the Purkinje cell layer or axial white matter. In addition, to evaluate Purkinje axon growth in different portions of the granular layer, the selected area was further subdivided into three domains, named "a," "b," and "c," corresponding to the superficial, medium, and deep regions of the granular layer, respectively (see the diagram in Fig. 3E). All of the Purkinje axon profiles present within the selected area were reproduced by Neurolucida, which calculated their total length. In addition, the density of these neurites was estimated by counting the number of times they crossed a $25 \times 25 \mu \mathrm{m}$ grid superimposed on the examined area. From each animal, three areas from distinct midsagittal sections were evaluated. Adjacent sections, stained by $2 \mathrm{~B} 6$ antibodies, were examined to check for the effects of enzyme or vehicle injection on CSPGs. Five animals per group (vehicle and ChABCinjected) were analyzed at each survival time, except at $7 \mathrm{~d}$, when six animals per group were considered. Finally, similar procedures were applied to assess the effects of CSPG degradation on axon myelination. In this case, MBP-positive profiles in the granular layer were quantified in three animals per group at $7 \mathrm{~d}$ survival. For all of these evaluations, 
statistical analysis was performed with the Student's $t$ test. In all instances, $p<0.05$ was considered statistically significant.

\section{Results}

\section{Distribution of CSPGs in the adult} rat cerebellum

The main goal of this study was to investigate the role played by CSPGs in the regulation of Purkinje axon plasticity in the adult cerebellar cortex. As a first step, we examined the distribution of CSPGs in the adult cerebellum using histochemical staining with the lectin WFA (Fig. $1 A-E$ ), which binds to the GAG chains of different types of CSPGs (Hartig et al., 1992). In line with previous reports (Seeger et al., 1994; Yamada et al., 1997), WFA staining was the most intense in the granular layer, which displayed a latex-like labeling pattern, with patches of different intensity and prominent pericellular nets around Golgi neurons (Fig. 1A,B). The terminal branches of the Purkinje axon infraganglionic plexus, visualized by anti-calbindin or anti-parvalbumin antibodies, were clearly associated with a WFA-positive matrix (Fig. $1 E$ ). In contrast, the molecular layer showed a diffuse labeling just above background level (Fig. 1A), and even the strong reaction product that decorated the dendrites of Golgi neurons abruptly faded at the granular-molecular layer interface (Fig. 1C,D). The aligned Purkinje cell bodies did not show clear pericellular nets, although they were somewhat highlighted by the different labeling pattern of the adjacent cortical layers (Fig. 1A). Finally, intense staining was present along the lobule white matter and also around the cell bodies and main dendrites of deep nuclei neurons (data not shown).

\section{ChABC-induced degradation of CSPGs} To obtain the degradation of CSPGs, we injected ChABC in the parenchyma of different vermal lobules. The effect of enzyme application was already achieved at $1 \mathrm{~d}$ after treatment. WFA labeling was almost completely absent in the granular layer and conspicuously reduced in the white matter of large cortical areas, usually encompassing lobules V-VII (Fig. $1 F)$. The same regions were also intensely immunolabeled with the antibody 2B6 (Fig. 1G), which recognizes the sugar moieties exposed after ChABC degradation of chondroitin sulfate but does not recognize intact CSPGs (Moon et al., 2001). No such modifications were observed when sections that received vehicle injections were processed for WFA histochemistry or 2B6 immunostaining.

\section{Effects of CSPGs degradation on Purkinje axons}

Except for a restricted region around the injection sites, which was excluded from our analysis, in vehicle-treated animals, the morphology of Purkinje intracortical plexus was similar to that observed in intact cerebella and consistent with previous reports (Fig. 2A,C) (Ramón y Cajal, 1911; Palay and Chan-Palay, 1974). In calbindin-immunostained sections, the corticofugal Purkinje axon emerges form the basal pole of the cell body and runs straight across the granular layer toward the white matter. Along this route, the neurite buds one or two thin recurrent collateral branches that ascend toward the Purkinje cell layer, where they terminate in several varicose chains forming the infraganglionic and supraganglionic plexus (Ramón y Cajal, 1911). The latter, which is located in the deep molecular layer, is poorly developed in the rat and obscured in our samples by the thick network of Purkinje dendrites. Thus, our attention was focused on the infraganglionic plexus, which is strictly confined to the upper region of the granular layer, just below the aligned Purkinje cell somata. 

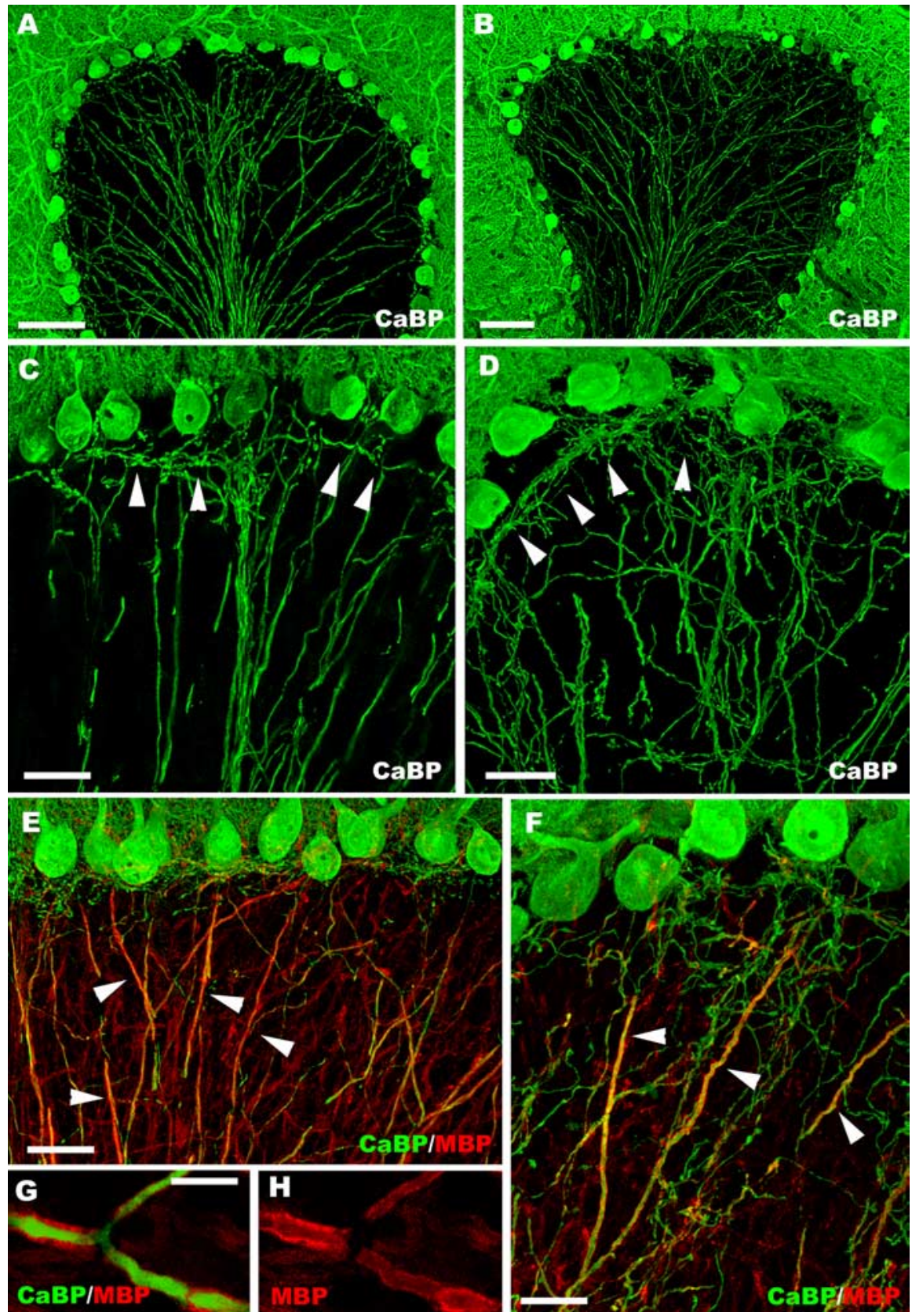

Figure 2. $\boldsymbol{A}-\boldsymbol{D}$, Morphology of Purkinje axons $7 \mathrm{~d}$ after ChABC injection. $\boldsymbol{A}, \boldsymbol{C}$, Confocal images of calbindin-immunostained sections from vehicle-injected animals. Note the typical distribution pattern of intracortical Purkinje axons with a thin infraganglionic plexus ( $\boldsymbol{C}$, arrowheads) confined within the upper granular layer. $\boldsymbol{B}, \boldsymbol{D}, \mathrm{ChABC}$-treated animals. Note the increased number of Purkinje axon profiles throughout the granular layer and the thickened infraganglionic plexus ( $\boldsymbol{D}$, arrowheads). $\boldsymbol{E}-\boldsymbol{H}$, Effect of CSPGs degradation on the axon-myelin relationships. Confocal images of cerebellar sections $7 \mathrm{~d}$ after ChABC injection, double immunostained for calbindin (green) and MBP (red). $\boldsymbol{E}, \boldsymbol{F}$, In both vehicle-injected $(\boldsymbol{E})$ and ChABC-treated cerebella $(\boldsymbol{F})$, corticofugal Purkinje axons (arrowheads), which run across the granular layer, maintain their myelin cover. $\boldsymbol{F}$, Note the numerous unmyelinated profiles extending into the deep granular layer of the $C h A B C$-injected cerebellum. The high-magnification pictures $\boldsymbol{G}$ and $\boldsymbol{H}$, also taken from an enzyme-treated cerebellum, show the origin of a recurrent Purkinje axon branch. Note that both the corticofugal axon and the thinner branch retain their myelin sheaths. CaBP, Calbindin. Scale bars: $\boldsymbol{A}, \boldsymbol{B}, 100 \mu \mathrm{m} ; \boldsymbol{C}-\boldsymbol{E}, 50 \mu \mathrm{m} ; \boldsymbol{F}$, $25 \mu \mathrm{m} ; \mathbf{G}, \boldsymbol{H}, 10 \mu \mathrm{m}$.

Although degradation of CSPGs was accomplished $1 \mathrm{~d}$ after ChABC application (see above), the regular arrangement of intracortical Purkinje axons was not overtly modified, indicating that axonal modifications do not initiate immediately after enzyme application. Most importantly, however, this observation shows that the higher amounts of neuritic profiles observed at longer survival times (see below) cannot be attributed to en- hanced penetration of the anti-calbindin antibody, induced by enzyme treatment. Indeed, the outgrowth of Purkinje axons was evident at $4 \mathrm{~d}$ and reached its maximum extension at $7 \mathrm{~d}$. By this time, there was a clear-cut increase of calbindinimmunolabeled profiles throughout the granular layer (Fig. 2B,D). The infraganglionic plexus was enlarged, appearing as a thick meshwork of varicose chains just below the Purkinje cell bodies. In addition, numerous terminal branches, with no preferential direction or orientation, covered the extent of the granular layer, although they appeared less dense in the deeper portions. The corticofugal axons, which could be followed in confocal stacks, did not show any dystrophic alterations such as torpedoes or thickened segments (Dusart and Sotelo, 1994; Rossi et al., 1994, 1995a), nor did they display the "hot-spots" with multiple sprouts, observed after application of anti-Nogo-A antibodies (Buffo et al., 2000).

The reorganization described thus far was quantified by comparing different morphometric parameters measured by means of the Neurolucida system in the granular layer of vehicle or enzymeinjected cerebella (Figs. $3 A-E, 4 A-D$ ). Consistent with our qualitative observations, $1 \mathrm{~d}$ after ChABC injection, the examined parameters (i.e., density and total length of Purkinje axons) (Fig. $3 A, B$ ) were not statistically different from the relevant control values (Student's $t$ test; density, $p=0.607$; total length, $p=0.524$ ). In contrast, both parameters were significantly higher in ChABC-treated rats at $4 \mathrm{~d}$ (Fig. $3 A, B$ ) (Student's $t$ test; density, $p=0.026$; total length, $p=0.049$ ), and the difference was further strengthened at $7 \mathrm{~d}$ (Fig. $3 A, B$ ) (Student's $t$ test; $p<0.001$ for both parameters).

Because the extent of growth in enzyme-injected cerebella seemed to be more pronounced in the upper portions of the granular layer, we examined the same morphometric parameters by subdividing this layer into three regions: superficial, medium, and deep, from the Purkinje cell layer to the white matter (corresponding to regions "a," "b," and "c," depicted in Fig. $3 E$ ). As shown in Figure 3, $C$ and $D$ (gray bars), in the vehicle-injected animals, at all time points, Purkinje axon profiles were always more numerous in the superficial region, in which the infraganglionic plexus is confined, whereas they resulted progressively less dense in the medium and deep regions. The same distribution pattern was essentially maintained in ChABC-treated cerebella (Fig. 3C,D, black bars). Nevertheless, although the values calculated at $1 \mathrm{~d}$ were not different from control, at 4 and $7 \mathrm{~d}$, both profile density (Fig. 3C) and total 
length (Fig. 3D) were significantly higher than the relevant control in upper and medium regions but not in the deep one abutting the axial white matter of the folium. On the whole, this analysis showed that the growth of Purkinje axons was more pronounced in the superficial portions of the granular layer and gradually subsided toward the white matter. Such a picture is consistent with the outgrowth from terminal branches of the infraganglionic plexus, which invade deeper regions of the granular layer.

\section{Effects of CSPGs degradation on} axon myelination

Injection of anti-Nogo-A antibodies in the cerebellar cortex induces sprouting from Purkinje axons in the granular layer (Buffo et al., 2000), and a similar phenomenon is observed after injury in GAP-43 (growthassociated protein-43)-overexpressing Purkinje cells (Buffo et al., 1997; Gianola and Rossi, 2004). In both conditions, in addition to the outgrowth of terminal fibers from the infraganglionic plexus, sprouting also occurs along the corticofugal neurite, and this phenomenon may be accompanied by disruption of the normal axon-myelin relationship (Gianola and Rossi, 2004).

To ask whether the outgrowth of Purkinje axons induced by ChABC application also involved disruption of their normal interactions with myelin sheaths, we compared vehicle and enzyme-injected cerebellar sections double labeled for calbindin and MBP at $7 \mathrm{~d}$ after treatment, at the peak of Purkinje axon sprouting. In both experimental groups, there were no clear differences from previous descriptions about myelination of Purkinje axons (Gianola et al., 2003; Gianola and Rossi, 2004; Clark et al., 2005). In control material (Fig. $2 E$ ), corticofugal axons were myelinated from a short distance from the cell body. Recurrent collaterals bud from a node of Ranvier along the intracortical course and were also covered by myelin sheaths, whereas the varicose chains of the infraganglionic plexus were devoid of myelin cover. The same features of corticofugal axons and ascending collaterals were also evident in ChABC-treated cerebella (Fig. $2 \mathrm{~F}-\mathrm{H}$ ). In these cases, however, the myelinated neurites running through the granular layer were surrounded by a dense meshwork of unmyelinated processes. These observations were confirmed by quantitative evaluation of the MBPpositive profiles in the granular layer, which yielded no significant differences between vehicle and enzyme-treated cere-
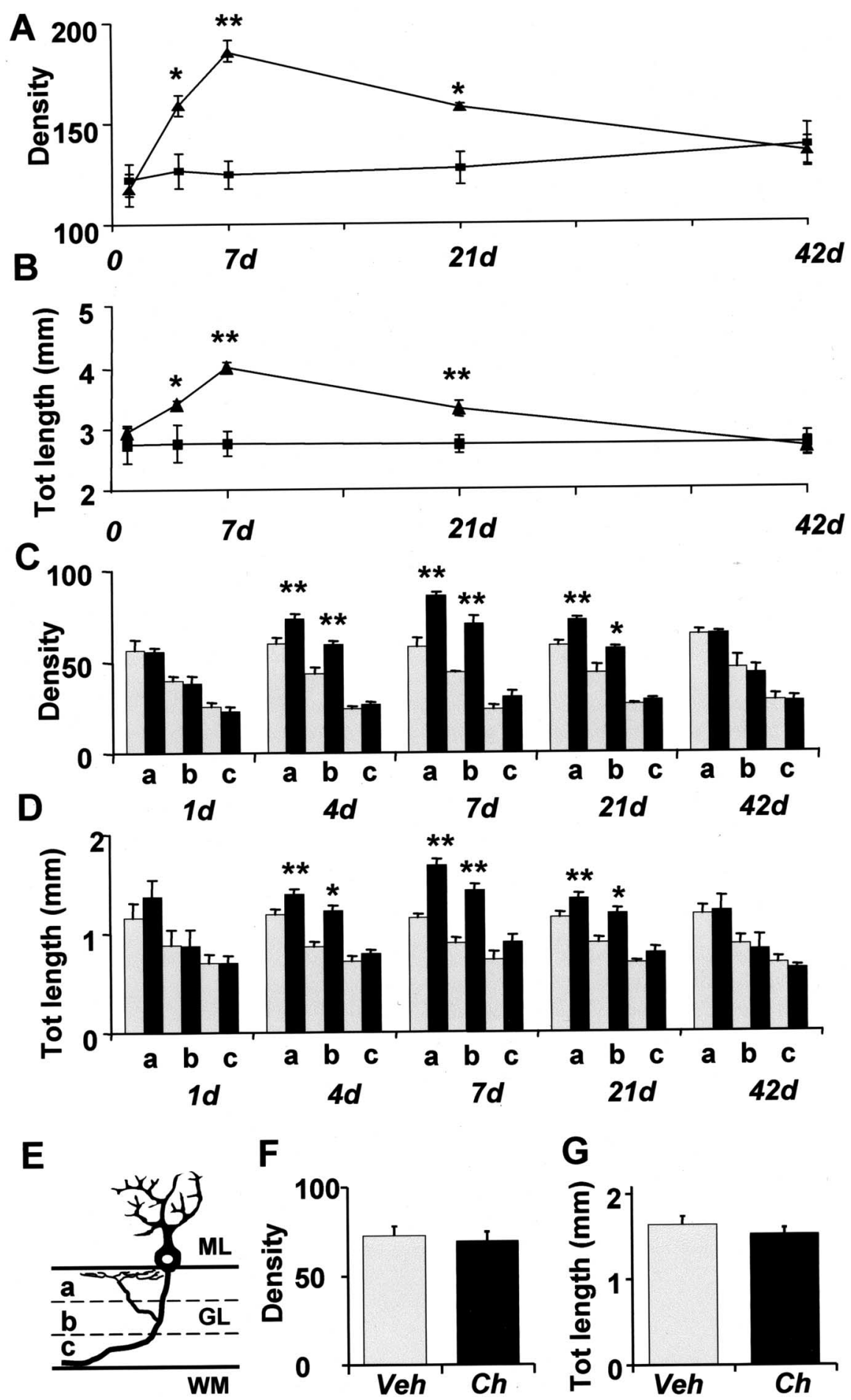

Figure 3. $\boldsymbol{A}-\boldsymbol{G}$, Quantification of the ChABC-induced remodeling of Purkinje axons. $\boldsymbol{A}, \boldsymbol{B}$, The time course of Purkinje axon sprouting after $\mathrm{ChABC}$ (triangles) or vehicle injection (squares). $\boldsymbol{A}, \boldsymbol{B}, \mathrm{A}$ significant increase of both profile density $(\boldsymbol{A})$ and total length $(\boldsymbol{B})$ is first evident at $4 \mathrm{~d}$, and the difference increases further at $7 \mathrm{~d}$. Thereafter, the values gradually decrease and return to control level at $42 \mathrm{~d}$. The same morphometric evaluations are shown in $\boldsymbol{C}$ and $\boldsymbol{D}$, calculated in the superficial (a), medium (b), and deep (c) regions of the granular layer (subdivision of the layer is depicted in $\boldsymbol{E}$ ). In the ChABC-treated animals (black bars; gray bars are vehicle), the density and total length of Purkinje axons are significantly increased at 4, 7, and $21 \mathrm{~d}$ in regions a and b but not in region c, supporting the conclusion that the sprouting processes are primarily sustained by terminal branches of the infraganglionic plexus. F, G, Quantification of myelinated axon profiles $7 \mathrm{~d}$ after ChABC injection. $\boldsymbol{F}, \mathbf{G}$, The histograms report the density $(\boldsymbol{F})$ and the total length $(\boldsymbol{G})$ of MBP-positive profiles in the granular layer of $\mathrm{ChABC}$ (black bars) or vehicle-injected (gray bars) animals. No significant differences were observed for any parameter. ML, Molecular layer; GL, granular layer; WM, white matter; Veh, vehicle; $\mathrm{Ch}, \mathrm{ChABC}$. All data are mean \pm SEM. Asterisks indicate values statistically different from time-matched controls: ${ }^{*} p<$ $0.05 ;{ }^{* *} p<0.01 ; n=5$ at each time point, for both vehicle- and enzyme-treated animals, except at $7 \mathrm{~d}$ when $n=6$. Error bars represent SEM. 

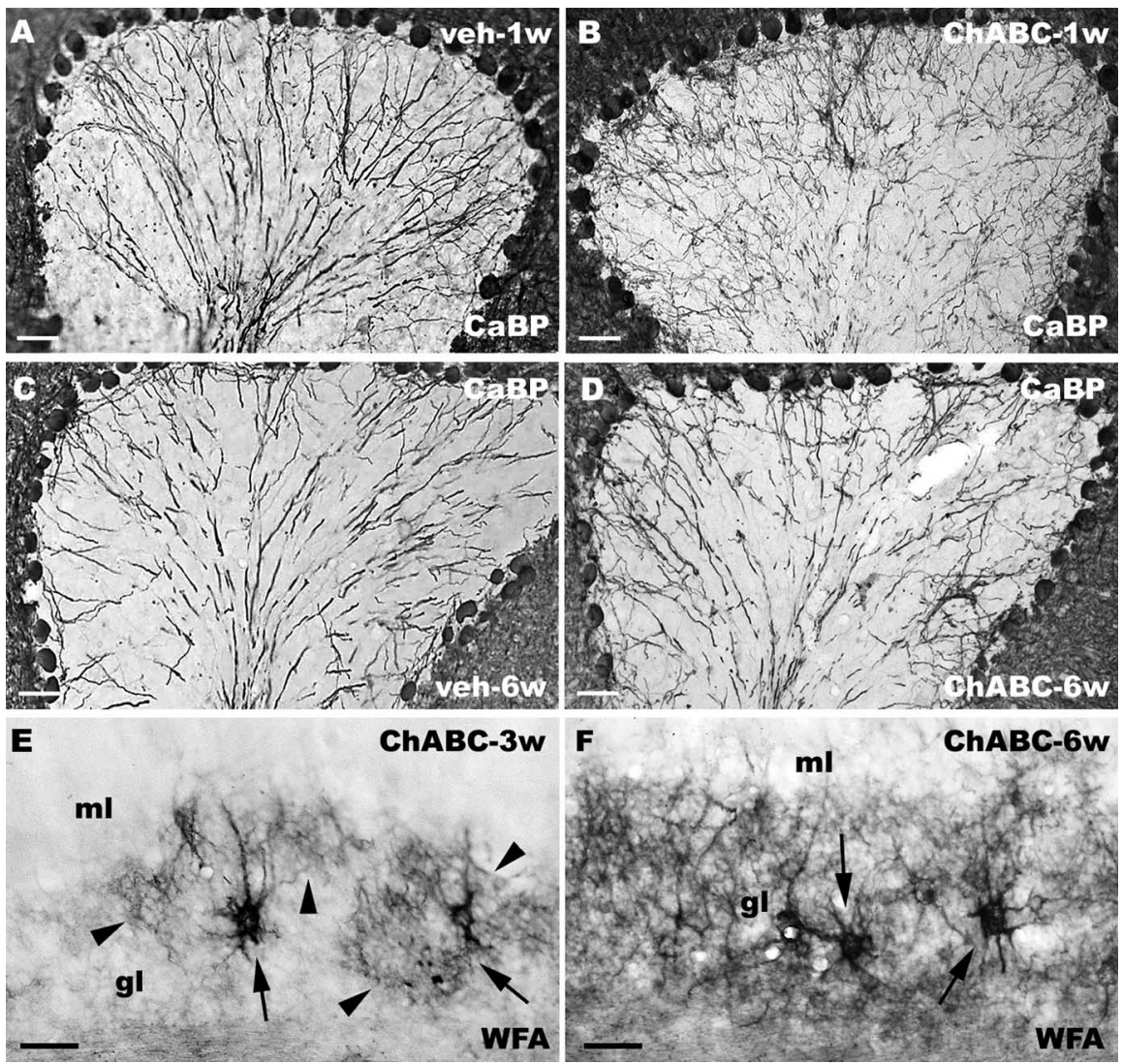

Figure 4. $\boldsymbol{A}-\boldsymbol{F}$, Recovery of ChABC-induced CSPGs degradation and Purkinje axon remodeling. $\boldsymbol{A}-\boldsymbol{D}$, Examples of $D A B /$ calbindin-immunolabeled sections used for quantitative analyses of Purkinje axon sprouting, $7 \mathrm{~d}(\boldsymbol{A}, \boldsymbol{B})$ or $42 \mathrm{~d}(\boldsymbol{C}, \boldsymbol{D})$ after vehicle $(\boldsymbol{A}, \boldsymbol{C})$ or ChABC $(\boldsymbol{B}, \boldsymbol{D})$ injection. $\boldsymbol{E}, \boldsymbol{F}$, Cerebellar sections labeled with WFA during the recovery period, 21 and $42 \mathrm{~d}$ after $\mathrm{ChABC}$ injection, respectively. Arrows point to perineuronal nets surrounding Golgi neurons. $\boldsymbol{E}$, At 3 weeks, the granular layer shows patches of labeled tissue (arrowheads) intermingled with unstained areas. $\boldsymbol{F}$, Full recovery of CSPG expression and distribution is achieved at 6 weeks. ml, Molecular layer; gl, granular layer; CaBP, calbindin. Scale bars, $50 \mu \mathrm{m}$.

bella (Fig. $3 F, G$ ) (Student's $t$ test; total length of MBP-positive profiles, $p=0.484$; density, $p=0.675)$. Although this quantitative estimate involved all of the myelinated axons running through the granular layer (i.e., also including mossy and climbing fiber axons), it shows that ChABC injection does not disrupt the normal relationship between Purkinje axons and myelin. Therefore, the outgrowth of Purkinje axons observed after CSPG degradation primarily consists of terminal branches of the infraganglionic plexus.

\section{Recovery of CSPG expression and axonal changes}

Our previous experiments indicate that the outgrowth of Purkinje axons induced by a single application of anti-Nogo-A antibodies is transitory, and normal conditions are reestablished within a few weeks (Buffo et al., 2000; Gianola et al., 2003). It has been shown that, after a single intraparenchymal injection of ChABC in the CNS, the normal content of CSPGs is reconstituted after several weeks (Brückner et al., 1998). Thus, we examined additional sets of animals at 21 and $42 \mathrm{~d}$ after enzyme application to determine whether the CSGPs had been restored and to assess whether this process was associated with retraction of Purkinje axons.

In WFA-labeled material $21 \mathrm{~d}$ after ChABC application, Golgi cells were again highlighted by strongly stained pericellular nets, whereas the surrounding granular layer displayed patches of labeled tissue with the typical latex-like pattern, intermingled with unstained areas (Fig. 3E). Analysis of calbindin-immunolabeled sections from these animals still revealed a conspicuous Purkinje axon sprouting in the granular layer. Morphometric evaluations showed that both profile density (Fig. 3A) and total length (Fig. $3 B$ ) were reduced compared with those calculated at $7 \mathrm{~d}$, but they were still significantly higher than their timematched controls (Student's $t$ test; density, $p=0.012$; total length, $p=0.006$ ). In addition, similar to the results obtained at 4 and $7 \mathrm{~d}$, statistically higher values were observed in upper and medium regions of the granular layer but not in the deepest one (Fig. 3C,D).

At $42 \mathrm{~d}$, both WFA histochemistry (Fig. $4 F$ ) and 2B6 immunolabeling of enzymetreated cerebella showed that the distribution pattern of CSPGs had been essentially reestablished. In addition, although some processes could still be encountered in the deep granular layer, the normal arrangement of intracortical Purkinje axons was essentially re-established. Morphometric evaluations did not show any significant difference from vehicle-treated cerebella in all regions of the granular layer (Fig. $3 A-D$ ) (Student's $t$ test; density, $p=0.828$; total length, $p=0.823$ ).

\section{Discussion}

To investigate the mechanisms that regulate neuritic growth and plasticity in the adult CNS, we examined the role played by CSPGs in maintaining the normal distribution pattern of Purkinje axons in the cerebellar cortex. Degradation of CSPGs, induced by a single application of ChABC, is followed by a vigorous outgrowth of intracortical Purkinje neurites, which invade the deeper regions of the granular layer. This phenomenon, which spontaneously regresses within several weeks, is not accompanied by overt alterations of the axon-myelin relationship, indicating that it is primarily sustained by sprouting from terminal branches of the infraganglionic plexus. These experiments show that removal of CSPGs, in the absence of injury or modifications of the external environment, is sufficient to induce axonal sprouting and point to the function of these molecules in preventing aberrant plasticity in the intact CNS.

\section{CSPGs regulate the structural plasticity of the Purkinje axon infraganglionic plexus}

After traumatic injury in the adult CNS, a major barrier to the regeneration of severed axons is the nonpermissive milieu created by glial scars. Several molecules of the extracellular matrix, including different types of CSPGs, are overexpressed at injury sites (McKeon et al., 1991; Levine, 1994; Lemons et al., 1999). Regeneration of transected axons (Moon et al., 2001; Bradbury et al., 2002), as well as compensatory reorganization of spared afferents (Tropea et al., 2003), can be significantly enhanced by enzymatic degradation of GAG chains. These findings identify CSPGs as negative factors that hamper repair processes in the injured CNS. However, similar to other regeneration-inhibitory mole- 
cules, CSPGs are constitutive elements of the extracellular matrix, expressed in normal conditions. This raises the question about their physiological role in the intact nervous tissue. Several lines of evidence indicate that CSPGs participate in different ontogenetic processes, including neurogenesis and gliogenesis (Brittis et al., 1992; Bandtlow and Zimmermann, 2000), cell migration (Bandtlow and Zimmermann, 2000), axon growth, and pathfinding (Snow et al., 1990; Jhaveri, 1993). In addition, formation of CSPG pericellular nets is associated with stabilization of synaptic contacts and closure of critical periods (Pizzorusso et al., 2002).

To ask whether CSPGs are involved in the maintenancespecific connection patterns in the adult cerebellum, we examined the Purkinje infraganglionic plexus. Despite their poor regenerative potential (Rossi et al., 1995b; Dusart et al., 1997; Carulli et al., 2004), Purkinje axons are capable of extensive sprouting at long-term after injury (Dusart and Sotelo, 1994; Dusart et al., 1999; Gianola and Rossi, 2002), when the scar environment acquires growth-promoting/permissive properties, including disappearance of CSPGs (Dusart et al., 1999, 2005; Morel et al., 2002). In addition, similar to other systems (Thallmair et al., 1998; Bareyre et al., 2002), sprouting from intact Purkinje axons, with aberrant growth into the deep granular layer, can be induced by application of anti-Nogo antibodies in both adult (Buffo et al., 2000) and developing (Gianola et al., 2003) cerebella. This suggests that Purkinje axons are endowed with strong inclination for structural plasticity that is normally restricted by inhibitory environmental cues (Rossi, 2004; Dusart et al., 2005). We show here that CSPGs also contribute to this regulation.

Previous studies have demonstrated that different types of CSPGs are expressed in the developing and adult cerebellum, with a highly precise localization in different cortical layers and white matter (Rauch et al., 1991; Friedlander et al., 1994; Grumet et al., 1994; Yamada et al., 1997). This distribution pattern correlates with that of several cell-adhesion molecules (Friedlander et al., 1994; Grumet et al., 1994), and it is spatiotemporally related to process growth and synaptogenesis (Rauch et al., 1991; Yamada et al., 1997; Tanaka et al., 2003). High levels of expression are maintained in adulthood, indicating that CSPG function continues after the end of development. Interestingly, in the adult cerebellum, the CSPG brevican is particularly enriched in glial sheaths that enwrap mossy fiber glomeruli, suggesting a specific role in maintaining the morpho-functional integrity of the synaptic complex (Yamada et al., 1997). Although a detailed analysis of CSPG types and distribution in the cerebellar cortex was beyond the scopes of this study, the clear association of WFA staining with varicose chains of the infraganglionic plexus shows that CSPGs in the cerebellar cortex are strategically positioned to regulate structural plasticity of different neuron populations, including Purkinje cells.

This idea was confirmed by the profuse sprouting of Purkinje axons occurring after ChABC application, similar to the growth induced by injection of anti-Nogo antibodies (Buffo et al., 2000). Although the growth phenomena observed in these two experimental conditions can be related to common underlying mechanisms (i.e., the removal of environmental inhibitory cues), which likely share common signaling pathways (Sandvig et al., 2004), some distinctive features characterize the axonal plasticity induced by $\mathrm{ChABC}$ application. First, in the present material, we failed to detect the thickened axon segments budding several short sprouts, which were frequently encountered after antiNogo injection (Buffo et al., 2000). Second, we found no evidence for myelin loss or alteration of the neurite-myelin relationship along corticofugal Purkinje axons and recurrent ramifications. Third, there was a conspicuous thickening of the infraganglionic plexus with numerous processes invading the deeper regions of the granular layer. Accordingly, quantitative evaluations revealed a clear gradient of Purkinje axon growth from superficial to deep regions of this layer. Together, these observations indicate that the plasticity of Purkinje axons induced by CSPG degradation does not involve the budding of new processes from the myelinated corticofugal neurites or collateral ramifications, but it is primarily sustained by sprouting from the terminal branches of the infraganglionic plexus. This suggests that different types of environmental cues act in a complementary manner. Myelinassociated proteins, which are present at both the axon-myelin interface and on the oligodendrocyte surface (Huber et al., 2002; Wang et al., 2002), stabilize the myelinated axon shaft and create a repulsive network for other incoming processes. CSPGs, distributed through the extracellular matrix, primarily contribute to the latter mechanism. However, because of their chemical heterogeneity and differential effect on distinct axon populations ( $\mathrm{Wu}$ et al., 2000; Heck et al., 2004), they may serve as a fine-tuning system to regulate neuritic plasticity in a cell type-specific manner.

\section{Dynamic regulation of neuritic growth in the adult CNS}

Similar to the application of anti-Nogo antibodies, the effects of ChABC injection were also transient. Consistent with observations in other systems (Brückner et al., 1998), several weeks after treatment, the 2B6 immunoreactivity faded out, whereas WFA staining recovered. The replacement of intact CSPGs was accompanied by retraction of the Purkinje axon sprouts from the deep regions of the granular layer. This suggests that the aberrant growth induced by CSPG degradation does not lead to the formation of stable synaptic contacts. This view is corroborated by previous observations on compensatory sprouting after partial denervation in the superior colliculus, in which the effect of CSPG removal is strong on process outgrowth but mild on the formation of new synapses (Tropea et al., 2003). Together, these findings indicate that, in the absence of specific external stimuli, the removal of inhibitory influences is not sufficient to induce the establishment of long-lasting connections. Indeed, in the adult visual cortex, changes in the pattern of ocular dominance only occur when CSPG degradation is associated with monocular deprivation, which alters the balance between the inputs from the two eyes (Pizzorusso et al., 2002). Hence, disruption of CSPG network creates permissive conditions for synaptic reorganization that must be driven by external stimuli.

In the adult nervous system, a fine equilibrium between growth-promoting and -inhibiting molecules is maintained to assure specific neuritic plasticity and synapse formation in response to environmental requirements. This homeostatic mechanism can be modulated by physiologic stimuli, as shown after salt loading in the supraoptic nucleus (Miyata et al., 2004), as well as pathologic manipulations, such as application of epileptogenic substances to the hippocampus (Heck et al., 2004). In these conditions, changes in the distribution pattern of different regulatory molecules, including CSPGs, trigger process outgrowth and synaptic reorganization that may result in functional adaptation or detrimental consequences, depending on the nature of the concomitant stimuli.

In the cerebellar cortex, these mechanisms are likely required to maintain the proper compartmentation of different axon populations within defined cortical domains. In this context, it is 
interesting to note that CSPG nets enwrap Golgi neurons in the granular layer but do not cover their dendrites in the molecular layer, which are contacted by parallel fibers (Palay and ChanPalay, 1974). This peculiar relationship suggests that CSPGs exert a local input-specific regulation, which can be different in distinct subcellular domains of the same neuron. A similar site-specific regulation might also apply for the infraganglionic and supraganglionic plexus of Purkinje axons that are developed in the granular and molecular layer, respectively. In this way, dynamic tuning of a wide repertoire of signaling molecules, and their cognate receptors, may allow selective plasticity of defined elements while preserving the overall organization of the cortical network. However, detailed analysis of the expression and function of these molecules in the cerebellar cortex is now needed to dissect the molecular mechanisms underlying these interactions.

\section{References}

Bandtlow CE, Zimmermann DR (2000) Proteoglycans in the developing brain: new conceptual insights for old proteins. Physiol Rev 80:1267-1290.

Bareyre FM, Haudenshild B, Schwab ME (2002) Long-lasting sprouting and gene expression changes induced by the monoclonal antibody IN-1 in the adult spinal cord. J Neurosci 22:7097-7110.

Bertolotto A, Manzardo E, Guglielmone R (1996) Immunohistochemical mapping of perineuronal nets containing chondroitin unsulfate proteoglycan in the rat central nervous system. Cell Tissue Res 283:283-295.

Bradbury EJ, Moon LD, Popat RJ, King VR, Bennett GS, Patel PN, Fawcett JW, McMahon SB (2002) Chondroitinase ABC promotes functional recovery after spinal cord injury. Nature 416:636-640.

Brakebusch C, Seidenbecher CI, Asztely F, Rauch U, Matthies H, Meyer H, Krug M, Bockers TM, Zhou X, Kreutz MR, Montag D, Gundelfinger ED, Fassler R (2002) Brevican-deficient mice display impaired hippocampal CA1 long-term potentiation but show no obvious deficits in learning and memory. Mol Cell Biol 22:7417-7427.

Brauer K, Härtig W, Bigl V, Brückner G (1993) Distribution of parvalbumin-containing neurons and lectin-binding perineuronal nets in the rat basal forebrain. Brain Res 631:167-170.

Brittis PA, Canning DR, Silver J (1992) Chondroitin sulfate as a regulator of neuronal patterning in the retina. Science 255:733-736.

Brückner G, Bringmann A, Hartig W, Koppe G, Delpech B, Brauer K (1998) Acute and long-lasting changes in extracellular-matrix chondroitinsulphate proteoglycans induced by injection of chondroitinase $\mathrm{ABC}$ in the adult rat brain. Exp Brain Res 121:300-310.

Buffo A, Holtmaat AJDG, Savio T, Verbeek S, Oberdick J, Oestreicher AB, Gispen WH, Verhaagen J, Rossi F, Strata P (1997) Targeted overexpression of the neurite growth-associated protein B-50/GAP-43 in cerebellar Purkinje cells induces sprouting in response to axotomy, but does not allow axon regeneration into growth permissive transplants. J Neurosci 17:8778-8791.

Buffo A, Zagrebelsky M, Huber AB, Skerra A, Schwab ME, Strata P, Rossi F (2000) Application of neutralizing antibodies against NI-35/250 myelinassociated neurite growth-inhibitory proteins to the adult cerebellum induces sprouting of uninjured Purkinje cell axons. J Neurosci 20:2275-2286.

Carulli D, Buffo A, Strata P (2004) Reparative mechanisms in the cerebellar cortex. Prog Neurobiol 72:373-398.

Caterson B, Christner JE, Baker JR, Couchman JR (1985) Production and characterization directed against connective tissue proteoglycans. Fed Proc 44:386-393.

Clark BV, Monsivais P, Branco T, London M, Häusser M (2005) The site of action potential initiation in cerebellar Purkinje neurons. Nat Neurosci 8:137-139.

Davies SJA, Fitch MT, Memberg SP, Hall AK, Raisman G, Silver J (1997) Regeneration of adult axons in white matter tracts of the central nervous system. Nature 390:680-683.

Dusart I, Sotelo C (1994) Lack of Purkinje cell loss in adult rat cerebellum following protracted axotomy: degenerative changes and reactive attempts of the severed axons. J Comp Neurol 347:211-232.

Dusart I, Airaksinen MS, Sotelo C (1997) Purkinje cell survival and axonal regeneration are age dependent: an in vitro study. J Neurosci 17:3710-3726.

Dusart I, Morel MP, Wehrlé R, Sotelo C (1999) Late axonal sprouting of injured Purkinje cells and its temporal correlation with permissive changes in the glial scar. J Comp Neurol 408:399-418.

Dusart I, Ghoumari A, Wehrlé R, Morel MP, Bouslama-Oueghlani L, Camand E, Sotelo C (2005) Cell death and axon regeneration of Purkinje cells after axotomy: challenges of classical hypotheses of axon regeneration. Brain Res Rev, in press.

Fawcett JW, Asher RA (1999) The glial scar and central nervous system repair. Brain Res Bull 49:377-391.

Filbin MT (2003) Myelin-associated inhibitors of axonal regeneration in the adult mammalian CNS. Nat Rev Neurosci 4:703-713.

Friedlander DR, Milev P, Karthikeyan L, Margolis RK, Margolis RU, Grumet M (1994) The neuronal chondroitin sulfate proteoglycan neurocan binds to the neural cell adhesion molecules Ng-CAM/L1/NILE and $\mathrm{N}-\mathrm{CAM}$, and inhibits neuronal adhesion and neurite outgrowth. J Cell Biol 125:669-680.

Gianola S, Rossi F (2002) Long-term injured Purkinje cells are competent for terminal arbour growth, but remain unable to sustain stem axon regeneration. Exp Neurol 176:25-40.

Gianola S, Rossi F (2004) GAP-43 overexpression in adult mouse Purkinje cells overrides myelin-derived inhibition of neurite growth. Eur J Neurosci 19:819-830

Gianola S, Savio T, Schwab ME, Rossi F (2003) Cell-autonomous mechanisms and myelin-associated factors contribute to the development of Purkinje axon intracortical plexus in the rat cerebellum. J Neurosci 23:4613-4624.

Grimpe B, Silver J (2002) The extracellular matrix in axon regeneration. Prog Brain Res 137:333-349.

Grumet M, Milev P, Sakurai T, Karthikeyan L, Bourdon M, Margolis RK, Margolis RU (1994) Interactions with tenascin and differential effects on cell adhesion of neurocan and phosphacan, two major chondroitin sulfate proteoglycans of nervous tissue. J Biol Chem 269:12142-12146.

Hartig W, Brauer K, Bruckner G (1992) Wisteria floribunda agglutininlabelled nets surround parvalbumin-containing neurons. NeuroReport 3:869-872.

Heck N, Garwood J, Loeffler JP, Larmet Y, Faissner A (2004) Differential upregulation of extracellular matrix molecules associated with the appearance of granule cell dispersion and mossy fiber sprouting during epileptogenesis in a murine model of temporal lobe epilepsy. Neuroscience 129:309-324.

Huber AB, Weinmann O, Brösamle, Oertle T, Schwab ME (2002) Patterns of Nogo mRNA and protein expression in the developing and adult rat and after CNS lesions. J Neurosci 22:3553-3567.

Jones LL, Tuszynski MH (2002) Spinal cord injury elicits expression of keratin sulfate proteoglycans by macrophages, reactive microglia, and oligodendrocyte progenitors. J Neurosci 22:4611-4624.

Jhaveri S (1993) Midline glia of the tectum: a barrier for developing retinal axons. Perspect Dev Neurobiol 1:237-243.

Lemons ML, Howland DR, Anderson DK (1999) Chondroitin sulfate proteoglycan immunoreactivity increases following spinal cord injury and transplantation. Exp Neurol 160:51-65.

Levine JM (1994) Increased expression of the NG2 chondroitin sulfate proteoglycan after brain injury. J Neurosci 14:4716-4730.

McKeon RJ, Schreiber RC, Rudge JS, Silver J (1991) Reduction of neurite outgrowth in a model of glial scarring following CNS injury is correlated with the expression of inhibitory molecules on reactive astrocytes. J Neurosci 11:3398-3411.

Miyata S, Akagi A, Hayashi N, Watanabe K, Oohira A (2004) Activitydependent regulation of a chondroitin sulfate proteoglycan 6B4 phosphacan/RPTPbeta in the hypothalamic supraoptic nucleus. Brain Res 1017:163-171.

Moon LD, Asher RA, Rhodes KE, Fawcett JW (2001) Regeneration of CNS axons back to their target following treatment of adult rat brain with chondroitinase ABC. Nat Neurosci 4:465-466.

Moreau-Fauvarque C, Kumanogoh A, Camand E, Jaillard C, Barbin G, Boquet I, Love C, Jones EY, Kikutani H, Lubetzki C, Dusart I, Chedotal A (2003) The transmembrane semaphorin Sema4D/CD100, an inhibitor of axonal growth, is expressed on oligodendrocytes and upregulated after CNS lesion. J Neurosci 23:9229-9239.

Morel MP, Dusart I, Sotelo C (2002) Sprouting of adult Purkinje cell axons 
in lesioned mouse cerebellum: "non-permissive" versus "permissive" environment. J Neurocytol 31:633-647.

Palay SL, Chan-Palay V (1974) Cerebellar cortex. Cytology and organization. New York: Springer.

Pizzorusso T, Medini P, Berardi N, Chierzi S, Fawcett JW, Maffei L (2002) Reactivation of ocular dominance plasticity in the adult visual cortex. Science 298:1187-1189.

Properzi D, Carulli D, Asher RA, Muir E, Camargo LM, van Kuppevelt TH, ten Dam GB, Furukawa Y, Mikami T, Sugahara K, Toida T, Geller HM, Fawcett JW (2005) Chondroitin 6-sulphate synthesis is up-regulated in injured CNS, induced by injury-related cytokines and enhanced in axongrowth inhibitory glia. Eur J Neurosci 21:378-390.

Ramón y Cajal S (1911) Histologie du système nerveux de l'homme et des vertébrés. Paris: Maloine.

Rauch U, Gao P, Janetzko A, Flaccus A, Hilgenberg L, Tekotte H, Margolis RK, Margolis RU (1991) Isolation and characterization of developmentally regulated chondroitin sulfate and chondroitin/keratan sulfate proteoglycans of brain identified with monoclonal antibodies. J Biol Chem 266:14785-14801.

Rossi F (2004) Regulation of the intrinsic growth properties in mammalian neurons. In: Brain damage and repair: from molecular research to clinical therapy (Delgado-Garcia JM, Herdegen T, Privat A, eds), pp 349-363. Amsterdam: Kluwer Academic Publishers.

Rossi F, Borsello T, Strata P (1994) Exposure to kainic acid mimics the effects of axotomy in cerebellar Purkinje cells of the adult rat. Eur J Neurosci 6:392-402.

Rossi F, Jankovski A, Sotelo C (1995a) Target neuron controls the integrity of afferent axon phenotype: a study on the Purkinje-cell climbing fibre system in cerebellar mutant mice. J Neurosci 15:2040-2056.

Rossi F, Jankovski A, Sotelo C (1995b) Differential regenerative response of Purkinje cell and inferior olivary axons confronted with embryonic grafts: environmental cues versus intrinsic neuronal determinants. J Comp Neurol 359:663-677.

Sandvig A, Berry M, Barrett LB, Butt A, Logan A (2004) Myelin-, reactive glia-, and scar-derived CNS axon growth inhibitors: expression, receptor signaling, and correlation with axon regeneration. Glia 46:225-251.

Schwab ME (2004) Nogo and axon regeneration. Curr Opin Neurobiol 14:118-124.

Seeger G, Brauer K, Härtig W, Brückner G (1994) Mapping of perineuronal nets in the rat brain stained by colloidal iron hydroxide histochemistry and lectin cytochemistry. Neuroscience 58:371-388.

Silver J, Miller JH (2004) Regeneration beyond the glial scar. Nat Rev Neurosci 5:146-156.

Snow DM, Steindler DA, Silver J (1990) Molecular and cellular characterization of the glial roof plate of the spinal cord and optic tectum: a possible role for a proteoglycan in the development of an axon barrier. Dev Biol 138:359-376

Tanaka M, Maeda N, Noda M, Marunouchi T (2003) A chondroitin sulfate proteoglycan PTP $\zeta / \mathrm{RPTP} \beta$ regulates the morphogenesis of Purkinje cell dendrites in the developing cerebellum. J Neurosci 23:2804-2814.

Thallmair M, Metz GAS, Z'Graggen WJ, Raineteau O, Kartje GL, Schwab ME (1998) Neurite growth inhibitors restrict plasticity and functional recovery following corticospinal tract lesions. Nat Neurosci 1:124-131.

Tropea D, Caleo M, Maffei L (2003) Synergistic effects of brain-derived neurotrophic factor and chondroitinase $\mathrm{ABC}$ on retinal fiber sprouting after denervation of the superior colliculus in adult rats. J Neurosci 23:7034-7044

Wang X, Chun S-J, Treloar H, Vartanian T, Greer CA, Strittmatter SM (2002) Localization of Nogo-A and Nogo-66 receptor proteins at sites of axon-myelin and synaptic contact. J Neurosci 22:5505-5515.

Wu YP, Siao CJ, Lu W, Sung TC, Frohman MA, Milev P, Bugge TH, Degen JL, Levine JM, Margolis RU, Tsirka SE (2000) The tissue plasminogen activator (tPA)/plasmin extracellular proteolytic system regulates seizureinduced hippocampal mossy fiber outgrowth through a proteoglycan substrate. J Cell Biol 148:1295-1304.

Yamada H, Fredette B, Shitara K, Hagihara K, Miura R, Ranscht B, et al (1997) The brain chondroitin sulfate proteoglycans brevican associates with astrocytes ensheathing cerebellar glomeruli and inhibits neurite outgrowth from granule neurons. J Neurosci 17:7784-7795. 